\title{
INCREMENTO EN MUTACIONES DE TIPO TRANSICIONAL PARA ATENUAR EL EFECTO DE SESGO EN LA INACTIVACIÓN DEL CROMOSOMA X CONDUCEN A LA APARICIÓN DE MUTACIONES CON EFECTO PATOLÓGICO EN EL GEN DE LA IDURONATO2 SULFATASA(IDS)(3.1.6.13)
}

\author{
TRANSITIONAL MUTATIONS INCREASE TO MITIGATE THE BIAS EFFECT ON THE X \\ CHROMOSOME INACTIVATION THAT LEADS TO THE ONSET OF MUTATIONS WITH \\ PATHOLOGICAL EFFECTS IN THE GENE FOR IDURONATO2 SULPHATASE (IDS)
}

$$
\text { (3.1.6.13) }
$$

Adalberto Sánchez Gómez ${ }^{1}$

${ }^{1 .}$ Departamento de Ciencias Fisiológicas. Escuela de Ciencias Básicas. Facultad de Salud. Universidad del Valle. Cali. Colombia.

Recibido: 20 de Noviembre de 2014

Aceptado: 10 de Diciembre de 2014

Correspondencia: Adalberto Sánchez Gómez, Departamento de Ciencias Fisiológicas. Escuela de Ciencias Básicas. Facultad de Salud. Universidad del Valle. Cali. Colombia. E-mail: adalberto.sanchez@correounivalle.edu.co

\begin{abstract}
RESUMEN
Varios autores han registrado la importancia de la metilación del gen IDS en el proceso de inactivación del cromosoma X. Adicionalmente, algunos pacientes con mucopolisacaridosis muestran un patrón de sesgo en la inactivación del cromosoma X y su relación con una disminución en la actividad de la iduronatosintasa 2. Nuestro estudio registra un patrón consistente de mutaciones de tipo transicional en este gen que podrían estar relacionadas con la génesis de mutaciones de tipo patológico, con el propósito de atenuar los efectos de una inactivación no aleatoria del cromosoma X. Se considera este hallazgo de particular importancia, ya que apoyaría la propuesta de un tamizaje de sitios de metilación en el cromosoma $\mathrm{X}$ en mujeres en edad fértil. Este tamizaje podría ayudar a disminuir las frecuencias alélicas de variantes relacionadas con un número variado de enfermedades asociadas al cromosoma X, como son: MPS tipo II, síndrome de Lesch-Nyhan y la distrofia muscular tipo Duchenne.
\end{abstract}

Palabras claves: Gen IDS, Mucopolisacaridosis, Mutaciones, Cromosoma X

\begin{abstract}
Several authors have reported the role of DNA methylation on IDS gene as a possible explanation of X chromosome inactivation. Besides, patients who suffer from mucopolysaccharidosis show a skewed X chromosome inactivation pattern related to a low or none iduronate-2-sulfatase activity. We reported a consisted pattern of transitional mutation c. $438 \mathrm{C}>\mathrm{T}$ on relative females of MPS II patients due to point mutations c.641C $>\mathrm{T}$, and c. $1122 \mathrm{C}>\mathrm{T}$. From several bioinformatics analysis we found an association between the inherited deamination mechanisms, responsible to avoid skewed $\mathrm{X}$ inactivation, and transitional mutations on pathogenic spots along IDS gene. Besides, data analysis predicted a possible increasing on pathogenic mutations on IDS gene. We consider this finding very valuable to propose a broad screening on fertile females of methylation target spots in X chromosome. Results from these screening test would help to prevent allele frequency increasing on X linked diseases as MPS, Lesch-Nyhan syndrome, and Duchenne muscular dystrophy.
\end{abstract}

Keywords: Gen IDS , Mucopolysaccharidosis, mutations , chromosome X 


\section{Introducción:}

La mucopilsacaridosis tipo II (MPS II o síndrome de Hunter; OMIM \#309900) es una enfermedad rara asociada el cromosoma sexual $\mathrm{X}$ en humanos y que se origina por alteraciones en las secuencias del gen de la iduronato 2 sulfatasa (IDS). Como menciona Pollard et al(1), cuando se examinan las alteraciones más frecuentes, se detecta un patrón preferencial en cambios de tipo transicional $\mathrm{C}>\mathrm{T}$. Adicionalmente, varios autores registran la presencia de un fenómeno de inactivación sesgada del cromosoma $\mathrm{X}$ en pacientes de MPS II como posible explicación a la aparición de la anomalía en mujeres. Este sesgo podría relacionarse con defectos de impronta en regiones que podrían ser blancos de metilaciones(2). En algunas ocasiones los rangos de sesgo llegan a relaciones extremas de 90:1, lo cual explicaría la presencia de la enfermedad en mujeres heterocigotas.

Recientemente, varios registros en la literatura plantean la aparición de mecanismos de reimpronta que permitan la activación de sitios de metilación de novo. Uno de estos mecanismos es la inducción de fenómenos transicionales tipo $\mathrm{C}>\mathrm{T}$, para permitir la acción de metilasas(3). De esta manera, se atenúa el efecto sesgo en la inactivación del cromosoma $\mathrm{X}$ y permitir un proceso de tipo aleatorio $(4,5)$.

Este trabajo registra el hallazgo de mutaciones de tipo transicional $\mathrm{C}>\mathrm{T}$ a lo largo del gen IDS, en un grupo de familias del suroccidente de Colombia, que estarían vinculados a procesos de reimpronta por deaminación para inactivar el cromosoma $\mathrm{X}$ y que explicarían la aparición de novo de mutaciones patogénicas en la población sin riesgo por descendencia. La confirmación de esta hipótesis podría conducir a la implementación de un programa de tamizaje en mujeres fértiles que ayudaría a disminuir la incidencia de enfermedades de tipo hereditario asociadas al cromosoma $\mathrm{X}$

\section{Materiales y métodos:}

El autor manifiesta que la propuesta metodológica fue revisada y aprobada por el comité de ética humana de la facultad de salud de la universidad del Valle.

Muestras: Un grupo de 8 familias compuestas por 12 individuos afectados con MPS II fueron sometidos a punción digital para la toma de sangre periférica sobre papel filtro. Este papel fue empacado para su envío al laboratorio Centogene en Rostock, Alemania, para el aislamiento de ADN, la secuenciación del gen de la IDS y el ensayo fluorométrico de la actividad enzimática. Los participantes de este estudio firmaron un formato de consentimiento informado, donde autorizaron el procedimiento.

Análisis de variantes genéticas: Los registros de variantes secuenciales registradas en la población fueron consignadas en tablas de frecuencia para su análisis poblacional. Para efectos de establecer un posible origen de estas mutaciones se utilizó el paquete de cómputo MEGA v 6.0. y para análisis estadísticos el paquete SPSS V 12.0.

Análisis de asociación patológica: Para variantes que no están registradas en el OMIM, se realizó un análisis mediante el uso de la plataforma para la predicción para efectos funcionales de SNPs humanos PolyPhen-2. Esta plataforma es abierta y gratuita (http://genetics.bwh.harvard.edu/pph2/).

\section{Resultados y Discusión:}

Las variantes secuenciales registradas en el estudio se consignan en la tabla No. 1, se nota una prevalencia de mutaciones de tipo transicional $\mathrm{C}>\mathrm{T}$ lo cual es consistente con lo informado por Pollard et al(1) para esta región génica

Tabla No. 1 Variantes secuenciales encontradas en la población de estudio

\begin{tabular}{|c|c|c|c|c|}
\hline $\begin{array}{c}\text { Region } \\
\text { Genómica }\end{array}$ & Posición & $\begin{array}{l}\text { Cambio } \\
\text { Proteíco }\end{array}$ & Ref. NCBI & $\begin{array}{l}\text { Asociación- } \\
\text { Patológica }\end{array}$ \\
\hline Exón 4 & c. $438 \mathrm{C}>\mathrm{T}$ & p.T146 T & rs1141608 & SNP \\
\hline Exón 4 & c.566dupT & $\begin{array}{l}\text { p.D190Gf } \\
\text { s*9 }\end{array}$ & $\begin{array}{l}\text { No regis- } \\
\text { trada }\end{array}$ & \\
\hline Exón 5 & c. $641 \mathrm{C}>\mathrm{T}$ & p.T214M & rs61736892 & Neutral \\
\hline Intrón 7 & $\begin{array}{l}\text { c. } 1007- \\
100 \mathrm{C}>\mathrm{T}\end{array}$ & N/A & rs116539145 & Neutral \\
\hline Exón 8 & c. $1122 \mathrm{C}>\mathrm{T}$ & p.G374G & $\begin{array}{l}\text { Rathmann, } \\
1996\end{array}$ & Patogénica \\
\hline
\end{tabular}

Cuando se examinó la distribución de estas mutaciones, se encontró una relación entre madres e hijos afectados, donde en las primeras se registra la presencia de la mutación c.438C $>\mathrm{T}$ y en los hijos afectados esta mutación en adición a las restantes transiciones. Este fenómeno podría ayudar a explicar el origen de mutaciones patogénicas como la c. $1122 \mathrm{C}>\mathrm{T}$, a través de un efecto de expansión de mutaciones transicionales, como mecanismo que evite el sesgo de inactivación del cromosoma $\mathrm{X}$ en las madres mediante la aparición de sitios de metilación de novo y de esta manera se prevenga las manifestaciones de la MPS 
II. Probablemente, estas madres posean una actividad de desaminación regulada durante el desarrollo embrionario, en donde la inactivación del cromosoma X sufre dos oleadas de selección: una primera por improntas particulares en zonas de metilación y una segunda de tipo aleatorio(6). A través de este mecanismo, se lograría reprogramar zonas de inactivación de tal manera que se patrocine la aleatoriedad del proceso evitando sesgos de inactivación que favorezcan la actividad de cromosomas con variantes génicas de tipo patológico (figura 1)
El registro de mutaciones transicionales de novo tipo $\mathrm{C}>\mathrm{T}$, como alternativa para la reimpronta de una inactivación aleatoria del cromosoma X, estaría involucrada en la aparición de nuevas variantes génicas de tipo patológico, como es el caso de estas mutaciones en el gen IDS y su relación con la MPS II. Su tamizaje tendría valor en programas de salud pública que pretendan disminuir la incidencia de enfermedades de tipo hereditario ligadas al sexo. Estos tamizajes deberán evaluar no solo la presencia de las mutaciones de tipo patológico, sino también las que no tengan efec-
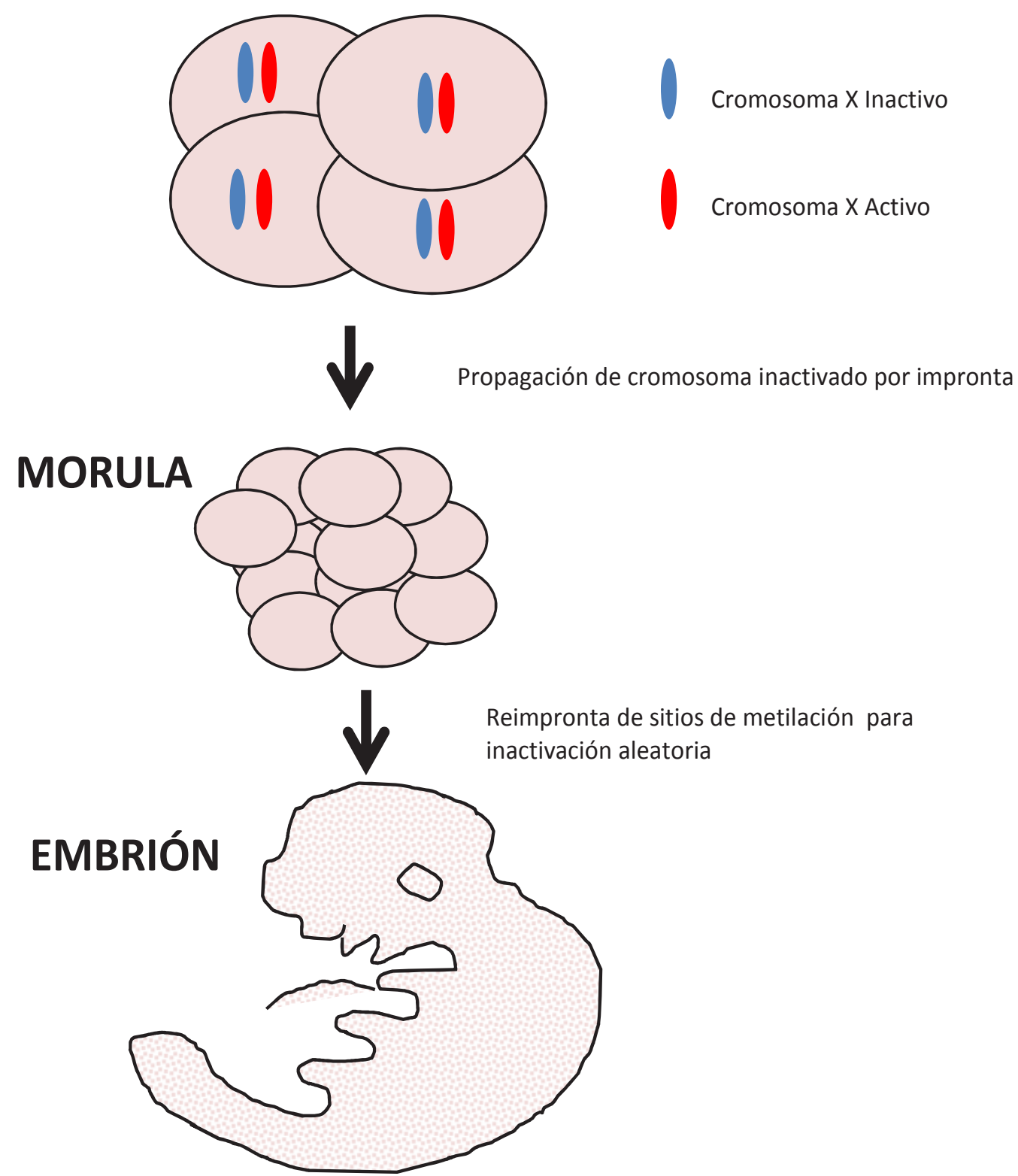

Figura No. 1 Alternancia en los procesos de inactivación del cromosoma $X$, trae como resultado un embrión mosaico con una tasa sin sesgo. 
to, como posibles predictores de fenómenos patológicos en generaciones futuras.

\section{Conclusiones:}

En este trabajo se registra una tendencia, en términos poblacionales de fijación de mutaciones transicionales $\mathrm{C} / \mathrm{T}$ para el gen de la iduronato sulfatasa, en el suroccidente colombiano. Esta tendencia podría estar relacionada con mecanismos de compensación de sesgos en la inactivación del cromosoma $\mathrm{X}$ en humanos, como se ha observado en otros estudios. Finalmente, se propone que la reimpronta del cromosoma $\mathrm{X}$ en familias del suroccidente colombiano puede con- vertirse en una variable de riesgo para el aumento de mutaciones patogénicas a lo largo del cromosoma X, que estarían afectando la incidencia de enfermedades ligadas al cromosoma X.

\section{Agradecimientos:}

El autor manifiesta sus agradecimientos al grupo de pacientes y sus familias que colaboraron en este estudio, así como también a los integrantes del grupo Laboratorio de Biología Molecular y Patogénesis de la Universidad del Valle, quienes realizaron sus sugerencias en el mismo.

\section{BIBLIOGRAFIA}

1. Pollard, Laura M., Jones, Julia R., and Wood, Tim C. 2013. Molecular characterization of 355 mucopolysaccharidosis patients reveals 104 novel mutations. J Inherit Metab Dis., (36),179187.

2. Piña-Aguilar, Raul E., Zaragoza-Arévalo, Gerardo R., Rau, Isabella, Gal, Andreas, AlcántaraOrtigoza, Miguel A., López-Martínez, Mónica S., and Santillán-Hernández, Yuritzi. (2013). Mucopolysaccharidosis type II in a female carrying heterozygous stop mutation of iduronate2 -sulfatase gene and showing a skewed chromosome inactivation. European Journal of Medical Genetics, (56),159-162.

3. Carrel, Laura, and Willard, Huntington F. (2005). X-inactivation profile reveals extensive variability in X-linked gene expression in females. Nature, (434),400-404.

4. Disteche, Christine M. (2012). Dosage Compensation of the Sex Chromosomes. Annu. Rev. Gene., (46),537-60.

5. Fortunato Lonardo, Paola Di Natale, Susanna Lualdi, Fabio Acquaviva, Cristina Cuoco, Francesca Scarano, Marianna Maioli, Luigi Michele Pavone, Grazia Di Gregorio, Mirella Filocamo, and Gioacchino Scarano. (2014). Mucopolysaccharidosis Type II in a Female Patient with a Reciprocal X;9 Translocation and Skewed X Chromosome Inactivation. Am. J. Med. Gen., (10), 2627-2632.

6. Payer, Bernhard, and Lee, Jeannie T. (2008). X Chromosome Dosage Compensation: How Mammals Keep the Balance. Annu. Rev. Genet., (42),733-72. 